\title{
Comparison of Femoral Triangle Block in Combination with IPACK to Local Periarticular Injection in Total Knee Arthroplasty
}

\author{
Xiang Li, MD ${ }^{1, *(1)}$ Yuanyuan Wang, MD²,* Yancheng Chen, MD ${ }^{1, *}$ Jiatian Qian, MD ${ }^{1}$ Shiao Li, MD ${ }^{1}$ \\ Song Chen, $\mathrm{PhD}^{3}$ Peiliang Fu, $\mathrm{PhD}^{1}$ \\ ${ }^{1}$ Department of Orthopedics, Naval Medical University, Changzheng \\ Hospital, Shanghai, China \\ ${ }^{2}$ School of Life Sciences, Bengbu Medical College, Bengbu, Anhui, China \\ ${ }^{3}$ Department of Orthopedics, The General Hospital of Western \\ Theater Command, Chengdu, Sichuan, China \\ J Knee Surg 2023;36:605-612.

\begin{abstract}
Address for correspondence Peiliang Fu, PhD, Department of Orthopedics, Changzheng Hospital, No. 415 Fengyang Road, Huangpu District, Shanghai 200003, China
\end{abstract} \\ (e-mail: fupeiliang@163.com).
}

\section{Abstract}

\section{Keywords}

- total knee arthroplasty

- femoral triangle block

- interspace between the popliteal artery and the capsule of the posterior knee

- rehabilitation

- nerve block
Objective This article studied the efficacy of two different analgesic methods after unilateral primary total knee arthroplasty (TKA) to find an effective analgesic method. Methods A randomized, double-blind, placebo, parallel, and controlled study was performed to evaluate the benefits of combining the femoral triangle block (FTB) and the interspace between the popliteal artery and the capsule of the posterior knee (IPACK). Forty patients diagnosed with knee osteoarthritis and underwent unilateral primary TKA with FTB and IPACK were divided grouped into the experimental group, and 40 patients undergoing TKA with intra-articular cocktail analgesic mixture local injection were grouped into the control group. All patients received the patient-controlled anesthesia pump for analgesia at postoperative 48 hours. The main indexes were postoperative knee joint rest and activity pain (visual analog scale) and muscle strength of the affected limb; secondary indexes were anesthetic consumption, total morphine consumption, range of motion, and complications (such as postoperative nausea and vomiting [PONV]).

Results There was no significant difference in the general data of each treatment group. Compared with the conventional group, the quadriceps muscle strength of the combined FTB and IPACK group was higher with significant statistical differences after surgery $(p<0.05)$. At postoperative $2,6,12,24,48$, and 72 hours, active pain was better than in the conventional group $(p<0.05)$. Resting pain was significantly smaller than the traditional group only at postoperative $2,6,12$, and 48 hours $(p<0.05)$. Morphine consumption, anesthetics consumption, and hospitalization time were lower than the conventional group, the difference being statistically significant. There were no significant differences between the two groups in postoperative wound healing, infection incidence, blood pressure, heart rate, rash, respiratory depression, deep vein thrombosis, and urinary retention. There were also no significant differences in PONV $(p>0.05)$.

Conclusion Combining FTB and IPACK significantly increased the quadriceps muscle in patients, together with relieving early pain and reducing the amount of anesthetic consumption at different postoperative intervals.

Xiang Li, Yuanyuan Wang, and Yancheng Chen contributed equally to this work.

received

February 9, 2021

accepted after revision

November 16, 2021

article published online

February 10, 2022 (c) 2022. Thieme. All rights reserved.

Thieme Medical Publishers, Inc.,

333 Seventh Avenue, 18th Floor,

New York, NY 10001, USA
DOI https://doi.org/

10.1055/s-0041-1741395.

ISSN 1538-8506. 
Total knee arthroplasty (TKA) is a standard surgical method used to improve quality of life and exercise performance in patients with advanced osteoarthritis. ${ }^{1}$ Although TKA is usually accompanied by moderate to severe postoperative pain, pain control can be achieved through analgesic methods, such as traditional analgesia with nonsteroidal antiinflammatory drugs (NSAIDs) ${ }^{2}$ and weak or strong opioids. ${ }^{3,4}$ However, approximately $50 \%$ of patients still present with severe immediate postoperative pain. Inadequate analgesia will affect outcomes in patients with postoperative rehabilitation, satisfaction, and overall prognosis. ${ }^{5}$

Femoral nerve block is a commonly used clinical peripheral nerve block (PNB) method in patients undergoing TKA. ${ }^{6}$ Nevertheless, this method can potentially cause a movement block leading to instability of the body center of gravity during postoperative functional exercise, increasing patients' risk of falling. The femoral triangle block (FTB) can provide analgesia by blocking the branches of the saphenous, medial vastus, and medial femoral cutaneous nerves (MFCN) to anesthetize the anteromedial portion of the knee for postoperative pain relief without affecting the quadriceps muscle. ${ }^{7,8}$

Moreover, recent reports have described a new PNB method, an alternative to a tibial nerve block that involves injecting local anesthetics in the interspace between the popliteal artery and capsule of the posterior knee (IPACK). The IPACK block acts on the posterior knee muscles and nerves without affecting the common peroneal nerve ${ }^{9}$; further, it provides more effective analgesic effects for patients without interfering with muscle strength. ${ }^{7}$ Therefore, we hypothesized that the use of FTB in combination with IPACK block was a more effective analgesic strategy for patients following TKA as compared with local periarticular injection that would not compromise muscle strength. We conducted a prospective, double-blind, randomized, and control study to assess the clinical efficacy of FTB combined with IPACK for analgesia after TKA including three goals: (1) pain scores at rest and when active, (2) muscle strength and range of motion (ROM), and (3) postoperative complications.

\section{Materials and Methods}

\section{Study Design}

We selected patients with knee osteoarthritis who underwent primary unilateral TKA for this study. We included patients with the American Society of Anesthesiologists score ranging from 1 to 3 , aged younger than 80 years, and who weighed 45 to $70 \mathrm{~kg}$. We excluded patients with mental health disorders, alcohol and drug dependence, morphine and local anesthetic drug hypersensitivity history, severe hepatic and kidney function impairment, severe knee deformity, chronic opioids, and NSAID tolerance. A biostatistician blinded to this research process generated a random number sequence with simple random numbers, and all random number cards were sealed with envelopes. When a patient was enrolled, they were assigned to the experiment/control group based on the odd/even number. A research nurse implemented this procedure. Blind codes were released after the data analysis stage. The patients were randomly assigned to the experimental or control group. Patient general information, visual analog scale (VAS) score, and joint ROM were obtained pre- and postoperatively. On the day before surgery, the patients were administered oral celecoxib $200 \mathrm{mg}$ twice and low molecular weight heparin (LMWH) calcium injection 4,000 IU subcutaneously twice to prevent deep venous thrombosis (DVT). Two assistants who did not participate in patient care or surgery prepared the reagent cocktail solutions for the control and experimental groups. Both the research assistants and patients were blinded to the treatment group allocation.

\section{Population of Patients}

The study was approved by the appropriate institutional review board, and written informed consent was obtained from all patients. Among 127 prospective patients, all responded to our research notice where 20 refused to participate, 80 patients met study inclusion criteria, while 27 patients were excluded for various reasons listed in - Fig. 1 that summarized the study recruitment strategy.

\section{Analgesic Mixture Compositions}

The compositions of the nerve-block mixture are ropivacaine hydrochloride injection $20 \mathrm{~mL}(75 \mathrm{mg} / 10 \mathrm{~mL})$, and morphine $1 \mathrm{~mL}(10 \mathrm{mg} / 1 \mathrm{~mL})$. The above reagents were diluted with saline to $50 \mathrm{~mL}$.

The intra-articular cocktail analgesic mixture local injection consists of the compound betamethasone injection (depotoxin) $1 \mathrm{~mL}(7 \mathrm{mg} / 1 \mathrm{~mL})$, ropivacaine hydrochloride injection $20 \mathrm{~mL}(75 \mathrm{mg} / 10 \mathrm{~mL})$, and morphine $1 \mathrm{~mL}(10 \mathrm{mg} /$ $1 \mathrm{~mL}$ ). The above reagents were diluted with saline to $100 \mathrm{~mL}$.

\section{The Combined FTB and IPACK Block Group}

After induction of general anesthesia, FTB and IPACK blocks were performed on the operative extremity. FTB was performed at a point slightly cephalad to the mid-thigh, defined as half the distance between the anterior superior iliac spine and patella base. Under ultrasound guidance, a 16-gauge echo needle was introduced and advanced to a point near the femoral artery in the triangle-shaped compartment bounded by the sartorius, vastus medialis, and adductor magnus muscles. After negative aspiration for blood, $10 \mathrm{~mL}$ of the nerve block mixture was injected. ${ }^{7}$ For the IPACK block, the probe was placed in the popliteal fossa and the needle was inserted from the lateral aspect of the knee in a posterolateral to anteromedial direction. ${ }^{7,10}$ The needle tip was placed 1 to $2 \mathrm{~cm}$ beyond the lateral edge of the artery and $20 \mathrm{~mL}$ of nerve block mixture was injected. In here, the joint cavity was partially injected with the same cocktail solution to eliminate interference.

\section{Surgery}

The surgeries were performed by experienced surgeons under general anesthesia utilizing the medial parapatellar approach for all TKAs. All patients ( 80 knees) underwent primary TKA using cemented Genesis II prostheses. Posterior stabilized prosthesis (PS) prosthetic devices were used in all 


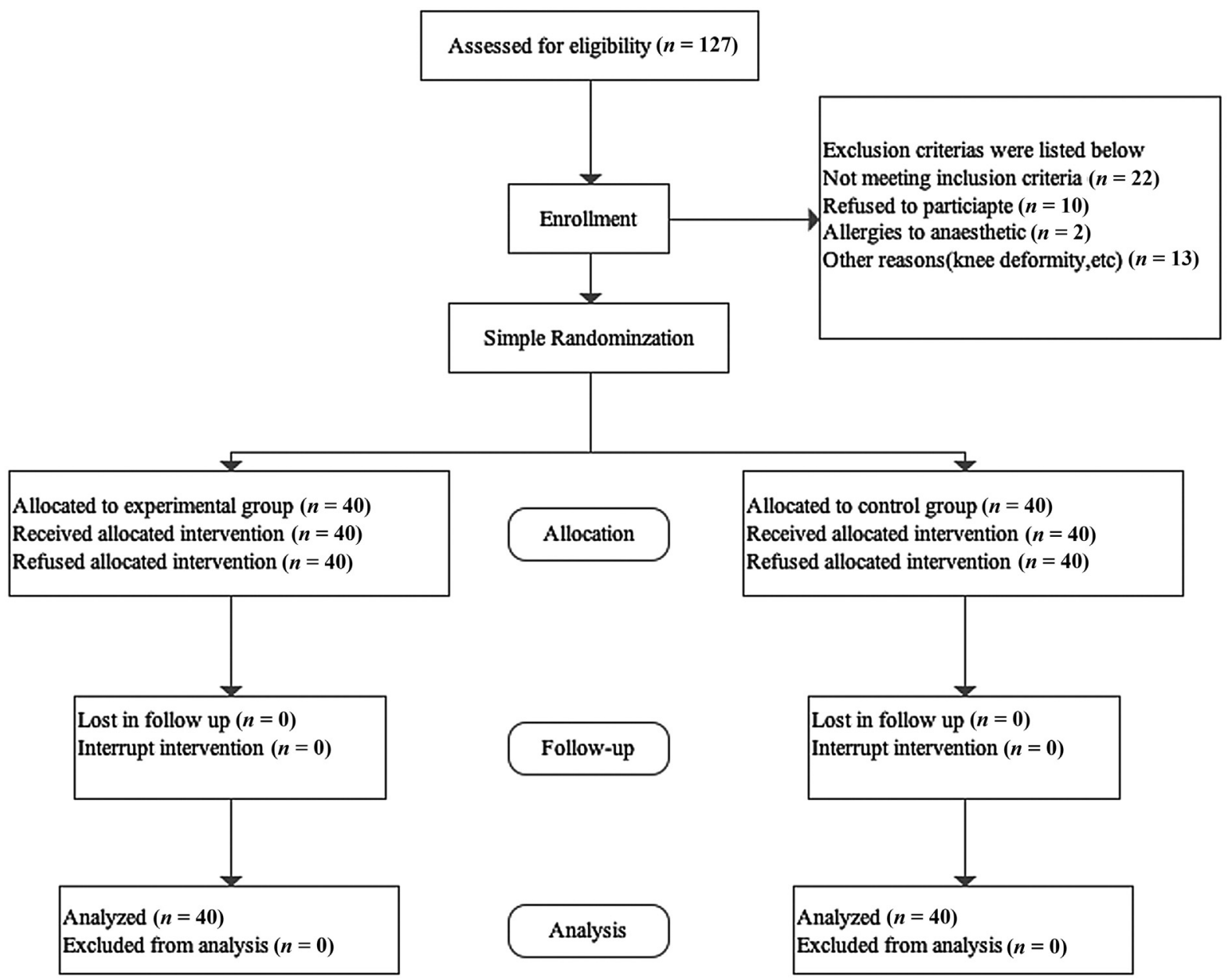

Fig. 1 The Consohdated Standards of Reporting Trials (CONSORT) flowchart of subject screening and enrollment.

knees. Traditional patellar denervation was performed in all patients using the cauterized peripatellar synovial membrane for peripatellar electrocauterization demulsification. A tourniquet was applied on all patients during this study for the entire procedure. We recorded intraoperatively surgical time and utility of lateral ligament release for balancing the TKA. The drainage tube was placed without patellar replacement.

\section{The Control Group}

Before prosthesis implantation, $20 \mathrm{~mL}$ of a compound anesthetic agent was injected into the posterior joint capsule, together with the medial and lateral collateral ligaments. ${ }^{11}$ Before closing the joint capsule, the surgeon injected $20 \mathrm{~mL}$ of the compound anesthetic agent into the quadriceps tendon, patellar tendon, and peripatellar joint capsule. ${ }^{11}$ The remaining $60 \mathrm{~mL}$ was injected into the synovium, subpatellar fat pad, medial and lateral support belt, and subperiosteal and subcutaneous tissues at multiple points without infiltrating the posterior capsule. ${ }^{11}$ The control group also used the same dose of physiological saline for nerve block.

\section{Postoperative Management}

All patients were treated with ice compression for 24 hours on postoperative day 1 and administered a COX-2 inhibitor celecoxib $200 \mathrm{mg}$ twice daily beginning from 6 hours postoperatively. Postoperative analgesia was administered using a patient-controlled anesthesia (PCA) pump with an LCP (loading dose ; continuous infusion ; PCA) model (load dosage $3 \mathrm{~mL}+$ lasting dosage $2 \mathrm{~mL} / \mathrm{h}+0.5 \mathrm{~mL}$ per time) within 48 hours. Twenty-five milligrams of morphine were diluted to $100 \mathrm{~mL}$ with normal saline in the PCA pump. When the PCA pump was stopped after 48 hours, morphine was administered intramuscularly (5-10 mg) according to the degree of pain (VAS score $>4$ ) and awareness of systemic conditions. LMWH (4,000 IU) was administered subcutaneously after surgery during the patients' hospital stay and lower extremity compression pumps were used to prevent DVT. After regaining consciousness, patients were encouraged to perform active straight-leg-raising movements and genuflexion exercises during postoperative recovery. Starting on postoperative day 1 , all patients received continuous passive motion machines with 30 degrees of motion for 2 weeks with a gradual increase of arc of motion dictated by the patient's pain tolerance. 


\section{Outcome Measures and Follow-Up}

Patient follow-up information and functional scores were collected, face-to-face or through phone calls, by the same experienced physician, blinded to the study group information.

Postoperative pain assessment mainly consisted of three aspects: (1) the assessment of resting and active VAS scores at 2, 6, 12, 24, 48, and 72 hours; (2) the measurement of lower limb muscle strength (quadriceps femoris, $\mathrm{N}$ ) by isokinetic training (CYBEX, America) at 2, 6, 12, 24, 48, and 72 hours; and (3) the ROM (degrees) at 2, 6, 12, 24, 48, and 72 hours.

Secondary outcomes included morphine consumption during each period (PCA basic dosage + PCA self-controlled dosage + intramuscular dosage), frequency of nauseas and vomiting, and dosing episodes of dorasilone given postoperatively. Discharge criteria consisted of a VAS score $<4$ and the ability to ambulate with assistance of crutches independent of a therapist. Patients were monitored until discharge for changes in blood pressure (BP), heart rate (HR), urinary retention, respiratory depression occurrence, drug allergy onset, and wound complications. Patient follow-up was scheduled to check for complications such as delayed infections at 1, 3, 6, and 12 months postoperatively and after that at 1 -year intervals.

\section{Statistical Analysis}

The analyses were performed with SPSS23.0 and Prism8 software. The classification data were expressed by frequency (\%). The chi-square test or Fisher's exact probability method was used for comparisons between groups; Enumeration data and measurement data were described by mean \pm standard deviation $(\mathrm{X} \pm S)$. Repeatedly measured data were analyzed by repeated measurement analysis of variance and Student's $t$-test for comparisons between groups. Quadriceps muscle strength, active pain VSA score, resting pain VAS score, and ROM were analyzed by repeated-measures analysis of variance. The Greenhouse-Geisser test was used to explore the within-subject effect. When the interaction was statistically significant, a simple effect analysis was performed. When the exchange was not statistically significant and the main result was statistically significant, the comparison was compared at each time within the group using the least significant difference method. A $p$-value of $<0.05$ was considered to be statistically significant.

\section{Result}

\section{General Information}

There was no difference in age distribution, sex ratio, body mass index, HR, and operation time between the two groups (-Table 1).

\section{Comparison of Active Pain and Resting Pain}

In terms of resting pain and active pain, the interaction effect of the two treatments and several time points were significant, respectively, $F(2,78)=868.99, p<0.001$, and $F(2$, $78)=895.21, p<0.001$. Based on these results, the following simple effects between time points and treatments were examined. A significant difference was observed in resting pain postoperatively up until the 12 -hour mark and after the 24-hour time point until 72 hours, in which no differences were observed only at 24-hour time point ( $\mathbf{- T a b l e ~} \mathbf{2}$ ).

Patients in the control group had significantly higher active pain scale measurements postoperatively at all time points observed ( - Table 3 ).

\section{Comparison of Quadriceps Muscle Strength and the Range of Motion}

Regarding quadriceps muscle strength, there was a significant interaction effect of the two treatments and several time points, $F(2,78)=6.90, p<0.001$. Therefore, based on these results, the simple effects of time points and treatments were examined. Quadriceps muscle strength was significantly higher in the experimental group early on after surgery and until 72 hours postoperatively ( - Table 4 ).

In terms of the ROM, there was a significant interaction effect of the two treatments and several time points, $F(2$, $78)=2611.93, p<0.001$. On the basis of the above results, the simple effects of time points and treatments were examined. There was significant improvement in postoperative knee ROM at the 48-and 72-hour time points (- Table 5).

Table 1 Demographics and surgical variables

\begin{tabular}{|l|l|l|l|l|}
\hline & Experimental group & Control group & $t$ value & $p$-Value \\
\hline Sample size $(n)$ & 40 & 40 & & \\
\hline Age $(\mathrm{y})$ & $67.82 \pm 7.50$ & $70.82 \pm 6.68$ & -1.89 & 0.06 \\
\hline Gender (M/F) & $15 / 25$ & $18 / 22$ & 0.46 & 0.50 \\
\hline Body mass index (BMI) & $25.41 \pm 2.39$ & $24.26 \pm 2.93$ & 1.92 & $>0.05$ \\
\hline HR (bpm) & $70.73 \pm 4.74$ & $70.80 \pm 4.65$ & -0.07 & 0.94 \\
\hline Operation time (min) & $98.60 \pm 10.23$ & $97.80 \pm 10.45$ & 0.35 & 0.73 \\
\hline Lateral release & $3(7.5 \%)$ & $4(10 \%)$ & & $1^{\text {a }}$ \\
\hline
\end{tabular}

Abbreviations: bpm, beats per minute; HR, heart rate.

Note: The Student's $t$-test and the chi-square test were used to compare continuous and categorical variables, respectively, between the two groups. Significance set at $p<0.05$.

${ }^{\mathrm{a}}$ Adopting Fisher's exact test. 
Table 2 The effect of different methods on resting pain

\begin{tabular}{|l|l|l|l|l|l|}
\hline & Experimental group & Control group & $(95 \% \mathrm{Cl})$ & $F$-Value & $p$-Value \\
\hline Sample size $(n)$ & 40 & 40 & & & \\
\hline Preop & $7.23 \pm 1.14$ & $7.50 \pm 0.78$ & -0.009 to 0.86 & 1.55 & 0.22 \\
\hline POH2 & $1.45 \pm 1.15$ & $3.82 \pm 1.94$ & 1.66 to 3.09 & 44.07 & $<0.001$ \\
\hline POH6 & $1.55 \pm 0.81$ & $2.83 \pm 1.81$ & 0.65 to 1.90 & 16.50 & $<0.001$ \\
\hline POH12 & $1.93 \pm 0.89$ & $2.83 \pm 1.95$ & 0.23 to 1.57 & 7.08 & 2.12 \\
\hline POH24 & $1.03 \pm 0.16$ & $1.13 \pm 0.40$ & -2.37 to 0.37 & 0.02 & 0.15 \\
\hline POH48 & $0.65 \pm 0.48$ & $0.93 \pm 0.57$ & 0.39 to 0.51 & 5.39 & 0.02 \\
\hline POH72 & $0.60 \pm 0.50$ & $0.83 \pm 0.39$ & 0.03 to 0.42 & 5.14 & 0.03 \\
\hline
\end{tabular}

Abbreviations: $\mathrm{Cl}$, confidence interval; $\mathrm{POH}$, postoperative hour; SD, standard deviation.

Note: Data are mean \pm SD and $95 \% \mathrm{Cl}$. The repeated-measures analysis of variance analyzed resting pain. Significance set at $p<0.05$.

Table 3 The effect of different methods on active pain

\begin{tabular}{|c|c|c|c|c|c|}
\hline & Experimental group & Control group & $(95 \% \mathrm{Cl})$ & $F$-Value & $p$-Value \\
\hline Sample size $(n)$ & 40 & 40 & & & \\
\hline Preop & $8.33 \pm 1.07$ & $8.75 \pm 0.87$ & -0.16 to 0.71 & 4.28 & 0.06 \\
\hline $\mathrm{POH} 2$ & $6.75 \pm 0.78$ & $7.65 \pm 0.74$ & 0.56 to 1.24 & 351.00 & $<0.001$ \\
\hline POH6 & $5.70 \pm 0.94$ & $5.7 \pm 0.70$ & -0.03 to 0.78 & 5.87 & $<0.02$ \\
\hline $\mathrm{POH} 12$ & $4.65 \pm 1.78$ & $6.33 \pm 1.16$ & 1.01 to 2.34 & 35.08 & $<0.001$ \\
\hline $\mathrm{POH} 24$ & $2.38 \pm 0.54$ & $3.9 \pm 0.86$ & 1.23 to 1.87 & 85.37 & $<0.001$ \\
\hline $\mathrm{POH} 48$ & $1.42 \pm 0.50$ & $2.30 \pm 0.52$ & 0.65 to 1.10 & 59.20 & $<0.001$ \\
\hline $\mathrm{POH} 72$ & $0.78 \pm 0.42$ & $1.48 \pm 0.51$ & 0.49 to 0.91 & 45.10 & $<0.001$ \\
\hline
\end{tabular}

Abbreviations: $\mathrm{Cl}$, confidence interval; $\mathrm{POH}$, postoperative hour; $\mathrm{SD}$, standard deviation.

Note: Data are mean \pm SD and $95 \% \mathrm{Cl}$. The repeated-measures analysis of variance analyzed active pain. Significance set at $p<0.05$.

Table 4 The influence of different methods on quadriceps muscle strength (N)

\begin{tabular}{|l|l|l|l|l|l|}
\hline & Experimental group & Control group & $(95 \% \mathrm{Cl})$ & $F$-Value & $p$-Value \\
\hline Sample size $(n)$ & 40 & 40 & & & \\
\hline Preop & $3.85 \pm 0.60$ & $3.76 \pm 0.59$ & -0.87 to 7.37 & 7.73 & 0.49 \\
\hline POH2 & $3.09 \pm 0.17$ & $2.64 \pm 0.20$ & -0.52 to -0.38 & 358.98 & $<0.001$ \\
\hline POH6 & $3.27 \pm 0.22$ & $3.02 \pm 0.14$ & -0.33 to -0.17 & 110.80 & $<0.001$ \\
\hline POH12 & $3.49 \pm 0.20$ & $3.20 \pm 0.13$ & -0.37 to -0.21 & 164.82 & $<0.001$ \\
\hline POH24 & $3.66 \pm 0.19$ & $3.40 \pm 0.12$ & -0.33 to -0.18 & 162.19 & $<0.001$ \\
\hline POH48 & $3.85 \pm 0.20$ & $3.61 \pm 0.14$ & -0.32 to -0.16 & 136.98 & $<0.001$ \\
\hline POH72 & $4.00 \pm 0.25$ & $3.66 \pm 0.13$ & -0.43 to -0.25 & 122.18 & $<0.001$ \\
\hline
\end{tabular}

Abbreviations: $\mathrm{Cl}$, confidence interval; $\mathrm{POH}$, postoperative hour; SD, standard deviation.

Note: Data are mean \pm SD and $95 \% \mathrm{Cl}$. The repeated-measures analysis of variance analyzed quadriceps muscle strength. Significance set at $p<0.05$.

\section{The Comparison of General Conditions and Complications after Surgery}

There were no significant differences in BP, HR, urinary retention, respiratory depression, rash, and DVT between the two groups (-Table 6). There were 15 patients in the experimental group and 18 patients in the control group subjected to nausea and vomiting with no significant differ- ence $(37.5 \%$ vs. $45 \%, p=0.50)$. Five patients in the experimental group and 13 patients in the control group each used morphine for analgesia following surgery. The utilization of intraoperative anesthetics and postoperative morphine for pain control as well as length of stay was significantly lower in the experimental group (-Table 6). Postoperative complications, including wound dehiscence and fat liquefaction, 
Comparison of Femoral Triangle Block in Combination with IPACK to Local Periarticular Injection in Total Knee

Table 5 The impact of different methods on the range of motion (ROM) (degrees)

\begin{tabular}{|l|l|l|l|l|l|}
\hline & Experimental group & Control group & $(95 \% \mathrm{Cl})$ & $F$-Value & $p$-Value \\
\hline Sample size $(n)$ & 40 & 40 & & & \\
\hline Preop & $107.50 \pm 10.19$ & $110.75 \pm 8.21$ & -0.87 to 7.37 & 2.877 & 0.10 \\
\hline POH2 & $19.88 \pm 6.75$ & $21.00 \pm 15.07$ & -4.07 to 6.32 & 1.74 & 0.67 \\
\hline POH6 & $33.38 \pm 7.79$ & $28.75 \pm 13.24$ & -9.46 to 0.21 & 4.01 & 0.05 \\
\hline POH12 & $34.88 \pm 6.75$ & $36.00 \pm 15.07$ & -4.07 to 6.32 & 0.17 & 0.68 \\
\hline POH24 & $43.38 \pm 7.7$ & $38.75 \pm 13.2$ & -9.46 to 0.21 & 4.01 & 0.05 \\
\hline POH48 & $53.38 \pm 9.09$ & $39.75 \pm 6.40$ & -17.12 to -10.13 & 60.98 & $<0.001$ \\
\hline POH72 & $62.13 \pm 8.16$ & $49.63 \pm 5.48$ & -15.59 to -9.41 & 59.45 & $<0.001$ \\
\hline
\end{tabular}

Abbreviations: $\mathrm{Cl}$, confidence interval; $\mathrm{POH}$, postoperative hour; SD, standard deviation.

Note: Data are mean \pm SD. The repeated-measures analysis of variance analyzed ROM. Significance set at $p<0.05$.

were observed in one patient in two cases in the experimental group and two in the control group, which were cured in the first stage after continual dressing changes. We did not observe any surgical site infections in either group during the follow-up period. In the control group, DVT was observed in one patient who reported in symptoms after treatment with antithrombotic therapy ( - Table 6 ).

\section{Discussion}

This study examined two different techniques for analgesia to alleviate knee joint pain and reduce the anesthetic influence on the quadriceps muscle in patients who underwent TKA. We found that FTB combined with IPACK block could reduce the anesthetic impact on muscle strength while providing adequate analgesia. Nevertheless, no clinical trials have been conducted to verify whether the combinations of FTB and PACK block could reduce postoperative complications and improve rehabilitation potential. Moreover, a prospective study that enrolled 120 patients who underwent unilateral TKA demonstrated that an adductor canal block (ACB) combined with IPACK block was a promising technique to improve middle-term postoperative pain management without affecting the motor function around the knee joint and increased patient walking distance compared with the use of ACB alone, ${ }^{12}$ which validated the notion that IPACK block did not influence muscle strength. The reasons for this might be manifold. Correspondingly, Kandarian et $\mathrm{al}^{13}$

Table 6 Postoperative metrics and complications

\begin{tabular}{|l|l|l|l|l|}
\hline & Experimental group & Control group & $t$-Value & $p$-Value \\
\hline Sample size ( $n)$ & 40 & 40 & & \\
\hline Systolic (mm Hg) & $134.55 \pm 11.05$ & $136.88 \pm 21.45$ & -0.61 & 0.55 \\
\hline Diastolic (mm Hg) & $73.70 \pm 9.70$ & $74.67 \pm 8.92$ & -0.47 & 0.64 \\
\hline HR (bpm) & $71.20 \pm 4.18$ & $72.13 \pm 6.87$ & -7.28 & 0.47 \\
\hline Hospitalization time (d) & $3.98 \pm 0.83$ & $5.00 \pm 0.75$ & -5.79 & $<0.001$ \\
\hline The consumption of anesthetics (propofol) (mL) & $42.63 \pm 5.43$ & $52.00 \pm 7.99$ & -6.14 & $<0.001$ \\
\hline Urinary retention & $1(2.5 \%)$ & $2(5.0 \%)$ & & $1^{\text {a }}$ \\
\hline Rash (Case) & $1(2.5 \%)$ & $1(2.5 \%)$ & & $1^{\text {a }}$ \\
\hline The consumption of morphine (mg) & $1.25 \pm 3.35$ & $3.25 \pm 4.74$ & -2.18 & 0.03 \\
\hline Wound complication (Case) & $2(5.0 \%)$ & $2(5.0 \%)$ & & $1^{\text {a }}$ \\
\hline Respiratory depression (Case) & $1(2.5 \%)$ & $2(5.0 \%)$ & & $1^{\text {a }}$ \\
\hline Nausea and vomiting (Case) & $15(37.5 \%)$ & $18(45 \%)$ & 0.46 & 0.50 \\
\hline DVT (Case) & 0 & $1(2.5 \%)$ & & 1 \\
\hline Intramuscular morphine (Case) & $5(12.5 \%)$ & $13(32.5 \%)$ & 4.59 & 0.03 \\
\hline
\end{tabular}

Abbreviations: bpm, beats per minute; DVT, deep vein thrombosis; HR, heart rate; SD, standard deviation.

Note: Data are mean \pm SD or number (\%) of patients. Binary categorical variables were analyzed by the chi-square test and Fisher's exact test. Continuous variables were analyzed using the Student's $t$-test. If the distribution of the variables was nonnormal, Wilcoxon's rank-sum test was analyzed adopted ( $z$-value). Significance set at $p<0.05$. Wound healing complications were observed at a mean of 5.5 (4-9) months of follow-up; all other events were observed during the hospitalization period.

${ }^{a}$ Adopting Fisher's exact test. 
retrospectively reviewed data from consecutive patients who underwent TKA by a single surgeon 4 months before and after IPACK block performance. They concluded that the addition of an IPACK block reduced postoperative pain, but not walking distance. Markedly, the combination of the FTB with the obturator nerve block technique was applied to 78 patients with significant postoperative pain following TKA and significantly alleviated postoperative pain without interfering with patient walking function, ${ }^{14}$ which suggested that FTB could also lessen the impact of muscle.

However, Berninger et $\mathrm{al}^{15}$ analyzed the impact of local infiltration analgesia (LIA), PNB, and general and spinal anesthesia on early functional recovery and pain control in primary unicompartmental knee arthroplasty, and had the opposite conclusion that there appeared to be a trend toward improved mobilization and muscle strength with general anesthesia and LIA, wherein general anesthesia had a greater tendency for mobilization amelioration. This was probably because general anesthesia and LIA were compared with sciatic nerve block, which had a pronounced effect on motor function in this study. ${ }^{13}$ It should be noted that this combination of anesthetics was more conducive to early postoperative functional recovery; however, the ROM did not improve significantly. This could probably be explained by the fact that this method might not reduce swelling, thus limiting the ROM.

The nerve blocks provided adequate analgesia postoperatively with lower activity and rest pain compared with the control group at numerous time points. Bjørn et $\mathrm{al}^{16}$ used the intermediate femoral cutaneous nerve (IFCN) or MFCN in combination with a proximal or distal FTB to investigate the area of cutaneous anesthesia concerning the surgical incision for the TKA and the anteromedial knee area. They found that anesthetizing the incision line after IFCN block (IFCNB) combined with proximal FTB produced a significantly higher anesthetic effect than that of the proximal FTB alone and distal FTB combined with IFCNB. Thobhani et al ${ }^{17}$ compared the effects of three regional techniques (femoral nerve catheter [FNC] block alone, FNC block combined with IPACK block, and ACB combined with IPACK block) on pain scores, opioid consumption, performance during physical therapy, and the duration of hospitalization in patients who underwent TKA. They affirmed that an IPACK block reduced opioid consumption by providing adequate supplemental analgesia following TKA compared with the FNC alone. The ACB combined with the IPACK block provided equivalent analgesia and improved physical therapy performance, leading to earlier hospital discharge. ${ }^{17}$

Furthermore, early postoperative active and resting pain were relieved in the FTB combined with the IPACK block group. On the contrary, weakened muscle strength decreased slightly with an increased turnover intention for early functional exercise. Exercise might promote the transport of inflammatory factors to the blood, thereby reducing the articular concentration of local inflammatory factors, ${ }^{18,19}$ which was beneficial for patients. This probably resulted in the further relief of resting and active pain. We found no significant between-group difference in 24-hour postoperative resting pain; however, the 48 -hour postoper- ative resting pain of patients in the FTB combined with the IPACK block group was significantly reduced than that of the control group. This was possibly because patients in the FTB combined with the IPACK block group had a delayed pain alleviation effect. The local anesthetic might have a weak diffusion ability in the distal femoral triangle area. ${ }^{9,16}$ However, nowadays, relevant research has lagged significantly. Perhaps, efforts can be geared toward making improvements along these lines. When FTB is implemented, the local anesthetic dose can be appropriately increased to increase local diffusion. If direct distribution of the local anesthetic to the popliteal fossa is not attained, many related nerves, such as the saphenous nerve, could possibly be blocked.

This study has some limitations that do not detract from our conclusions. First, we evaluated quadriceps muscle strength and knee ROM with the surgical bandage still intact, which potentially limits flexion and muscles strength. However, this protocol was applied symmetrically to both the experimental and control group which would limit bias. Furthermore, the short postoperative follow-up may not be adequate enough to observe complications related to the nerve block administered including but not limited to nerve injury, block site infection, and mechanical falls due to muscle weakness which could limit the impact of improved outcomes appreciated during the hospital course in the group that received the nerve block.

Compared with the control group, the combination of FTB and IPACK block significantly increases patient quadriceps muscle strength, relieved early postoperative pain, and reduced the amount of anesthetic consumption at different postoperative intervals. This new analgesic method could have good prospects in clinical application by attenuating postoperative pain, mitigating postoperative complications, and accelerating postoperative rehabilitation.

\section{Conflict of Interest}

None declared.

\section{References}

1 Yano T, Imaizumi T, Matsu-Ura H, Takahashi T. Relationship between dexamethasone added to periarticular anesthetic infiltration and postoperative nausea and vomiting following total knee arthroplasty under general anesthesia: a retrospective observational study. JA Clin Rep 2020;6(01):66

2 Davies AF, Segar EP, Murdoch J, Wright DE, Wilson IH. Epidural infusion or combined femoral and sciatic nerve blocks as perioperative analgesia for knee arthroplasty. Br J Anaesth 2004;93(03): 368-374

3 Sigirci A. Pain management in total knee arthroplasty by intraoperative local anesthetic application and one-shot femoral block. Indian J Orthop 2017;51(03):280-285

4 Korean KSKorean Knee Society. Guidelines for the management of postoperative pain after total knee arthroplasty. Knee Surg Relat Res 2012;24(04):201-207

5 Li JW, Ma YS, Xiao LK. Postoperative pain management in total knee arthroplasty. Orthop Surg 2019;11(05):755-761

6 Gao F, Ma J, Sun W, Guo W, Li Z, Wang W. Adductor canal block versus femoral nerve block for analgesia after total knee arthroplasty: a systematic review and meta-analysis. Clin J Pain 2017;33 (04):356-368 
7 Ohgoshi Y, Matsutani M, Kubo EN. Use of IPACK block with continuous femoral triangle block for total knee arthroplasty: a clinical experience. J Clin Anesth 2019;54:52-54

8 Lee HJ, Bach JR, DeLisa JA. Medial femoral cutaneous nerve conduction. Am J Phys Med Rehabil 1995;74(04):305-307

9 Runge C, Bjørn S, Jensen JM, et al. The analgesic effect of a popliteal plexus blockade after total knee arthroplasty: a feasibility study. Acta Anaesthesiol Scand 2018. Doi: 10.1111/aas.13145

10 Jung DW, Shon WY, Seo SS, Kim OG, Lee IS. Comparison of the postoperative analgesic effect for infiltration between the popliteal artery and the capsule of the posterior knee and that of periarticular multimodal drug injection in total knee arthroplasty: retrospective study in the immediate postoperative period. Knee Surg Relat Res 2020;32(01):1

11 Fu P, Wu Y, Wu H, Li X, Qian Q, Zhu Y. Efficacy of intra-articular cocktail analgesic injection in total knee arthroplasty - a randomized controlled trial. Knee 2009;16(04):280-284

12 Sankineani SR, Reddy ARC, Eachempati KK, Jangale A, Gurava Reddy AV. Comparison of adductor canal block and IPACK block (interspace between the popliteal artery and the capsule of the posterior knee) with adductor canal block alone after total knee arthroplasty: a prospective control trial on pain and knee function in immediate postoperative period. Eur J Orthop Surg Traumatol 2018;28(07):1391-1395

13 Kandarian B, Indelli PF, Sinha S, et al. Implementation of the IPACK (infiltration between the popliteal artery and capsule of the knee) block into a multimodal analgesic pathway for total knee replacement. Korean J Anesthesiol 2019;72(03):238-244

14 Runge C, Børglum J, Jensen JM, et al. The analgesic effect of obturator nerve block added to a femoral triangle block after total knee arthroplasty: a randomized controlled trial. Reg Anesth Pain Med 2016;41(04):445-451

15 Berninger MT, Friederichs J, Leidinger W, et al. Effect of local infiltration analgesia, peripheral nerve blocks, general and spinal anesthesia on early functional recovery and pain control in unicompartmental knee arthroplasty. BMC Musculoskelet Disord 2018;19(01):249

16 Bjørn S, Nielsen TD, Moriggl B, Hoermann R, Bendtsen TF. Anesthesia of the anterior femoral cutaneous nerves for total knee arthroplasty incision: randomized volunteer trial. Reg Anesth Pain Med 2019:rapm-2019-100904

17 Thobhani S, Scalercio L, Elliott CE, et al. Novel regional techniques for total knee arthroplasty promote reduced hospital length of stay: an analysis of 106 patients. Ochsner J 2017;17(03):233-238

18 Jules- Elysee KM, Wilfred SE, Memtsoudis SG, et al. Steroid modulation of cytokine release and desmosine levels in bilateral total knee replacement: a prospective, double-blind, randomized controlled trial. J Bone Joint Surg Am 2012;94(23):2120-2127

19 Si HB, Yang TM, Zeng Y, et al. Correlations between inflammatory cytokines, muscle damage markers and acute postoperative pain following primary total knee arthroplasty. BMC Musculoskelet Disord 2017;18(01):265 